\title{
Lesão por fricção e lesão por pressão em idosos: prática de enfermagem baseada em evidências
}

\author{
Juliana Balbinot Reis Girondi ${ }^{*}$, Scheila Monteiro Evaristo, Francisco Reis Tristão, \\ Lúcia Nazareth Amante, Luciara Fabiane Sebold, Milena Ronise Calegari
}

Universidade Federal de Santa Catarina, Florianópolis, SC, Brasil

\section{Histórico do Artigo \\ Recebido em: \\ 12/03/2021 \\ Aceito em: \\ $01 / 07 / 2021$}

\section{Palavras-chave:}

Enfermagem; lesão por pressão; fricção; idosos; enfermagem baseada em evidências

Keywords:

Nursing; pressure ulcer; friction; aged; evidencebased nursing

\begin{abstract}
RESUMO
O objetivo da pesquisa foi identificar estudos de intervenções preventivas, diagnósticas e de tratamento de Lesão por Fricção e Lesão por Pressão em idosos. Foi realizada uma revisão integrativa nas bases Base de Dados de Enfermagem, Latino-Americana e do Caribe em Ciências da Saúde, Cumulative Index to Nursing and Allied health literature; Medical Literature Analysis and Retrieval System Online e Scientific Eletronic Library Online, período entre 2014 e 2020. Os dados dos estudos foram organizados e apresentados em quadro e, o corpus de análise classificados em consonância ao nível de evidência de Melnyk e Fineout-Overholt. Foram identificados 42 artigos, onde apenas dois foram classificados como nível de evidência forte, agrupados em quatro categorias temáticas: Identificando fatores de risco para o desenvolvimento de lesões; Prevenção e manutenção da integridade da pele; diagnóstico de enfermagem nas lesões de pele e Tratando as Lesões por Fricção e por Pressão. As publicações estão voltadas para identificação e avaliação de riscos de Lesões por Pressão e, em menor quantitativo, em Lesões por Fricção. Os diagnósticos de enfermagem apresentam-se com maior carência de publicações, em ambos os tipos de lesões. Não foram identificados estudos sobre cuidados de enfermagem aos idosos com Lesão por Fricção. Conclusão: Torna-se necessário que novas pesquisas, no intuito de fortalecer e embasar a prática assistencial do enfermeiro pautada na clínica baseada em evidência científica.
\end{abstract}

Pressure injury and friction injury in elderly: evidence-based nursing practice

\section{ABSTRACT}

The aim of the study was to identify studies of preventive, diagnostic and treatment interventions for Friction Injury and Pressure Ulcer in the elderly, as well as the levels of scientific evidence. An Integrative review was performed in the Latin American and Caribbean Health Sciences Database, Cumulative Index to Nursing and Allied health literature; Medical Literature Analysis and Retrieval System Online and Scientific Electronic Library Online, period 2014-2020. The data from the studies were organized and presented in a table and the corpus of analysis was classified according to the evidence level of Melnyk and Fineout-Overholt. Forty-two articles were identified, two were classified as strong. The articles originated four thematic categories: Identifying risk factors for the development of lesions; Prevention of maintaining skin integrity; Nursing Diagnosis in Skin Lesions and Treating Friction and Pressure Ulcer. The publications focus on identifying and assessing risks for Pressure Ulcer and, to a lesser extent, for Friction Injuries. The nursing diagnosis has the greatest lack of publications in both cases, but no nursing care was identified for the elderly with friction injury. It is necessary that further studies be clarified in order to strengthen and support the practice of nursing care based on clinical evidence based.

\section{Introdução}

O envelhecimento populacional traz necessidades específicas, especialmente relacionadas aos cuidados em saúde, devido às diversas mudanças anatômicas e funcionais. Dentre elas, ressaltamos o tecido tegumentar que se torna frágil, apresentando piora quando exposto a fatores externos como fricção, contusão, pressão e cisalhamento,

\footnotetext{
* Autor correspondente: juliana.balbinot@ufsc.br (Girondi J.B.R.)
} 
fatores estes que estão ligados ao surgimento de Lesão por Fricção (LF) e Lesão por Pressão (LP) (1).

Neste sentido, cabe ao enfermeiro reorganizar e planejar constantemente o cuidado para prevenção de lesões de pele, orientando o idoso/cuidador a respeito da prevenção destas, e na identificação de riscos a fim de preservar o comprometimento da pele. Deste modo, considerando a importância desta ação na diminuição do impacto negativo na qualidade de vida do idoso assim como, seus cuidadores e familiares, justifica-se maior atenção do enfermeiro no atendimento à população idosa a fim de contribuir para redução de gastos com tratamentos de lesões evitáveis (2).

Nessa perspectiva reconhece-se a necessidade de conferir alicerces tangíveis a todo o conhecimento científico que ainda precisa ser construído acerca das LF e LP, recomendando e estimulando o desenvolvimento de estudos para elucidar a epidemiologia das LF e LP nos serviços de saúde, bem como ofertar as melhores práticas de cuidado de enfermagem.

Desse modo, a pesquisa objetivou identificar estudos que apresentem as intervenções preventivas, diagnósticas e de tratamento de LP e LF em idosos, bem como o grau de evidência científica dos estudos publicados.

\section{Método}

Este estudo trata-se de Revisão Integrativa (RI) elaborada por meio de protocolo de estudo, o qual respeita as seguintes etapas: elaboração da questão de estudo; estabelecimento dos critérios de inclusão e exclusão; identificação dos estudos selecionados; categorização e análise dos estudos selecionados, análise e interpretação dos resultados e apresentação da revisão e síntese do conhecimento (3).

Para realização dessa revisão selecionou-se a questão norteadora: quais são evidências científicas das intervenções preventivas, diagnósticas e de tratamento de LP e LF em idosos?

A pesquisa foi realizada em periódicos indexados nas bases de dados: Literatura LatinoAmericana e do Caribe em Ciências da Saúde (LILACS); Cumulative Index to Nursing and Allied health literature (CINAHL); Medical Literature Analysis and Retrieval System Online (PUBMED/MEDLINE) e Scientific Electronic Library Online (SciELO) por meio de estratégias de busca desenvolvidas especificamente para cada base. A base de dados Scopus foi excluída por não apresentar estudos elegíveis, assim como a base de dados em Enfermagem (BDENF), no qual apresentou dois estudos, sendo esses repetidos.

O processo foi desenvolvido com auxílio de bibliotecária experiente neste tipo de pesquisa. Para composição da estratégia de buscas e foram utilizados os descritores: Aged/aging/Senescence/Pressure Ulcer/Decubitus Ulcer/Bed Sore/Pressure Sore/Pressure Injury/Wound and Injury/Multiple Wound/Skin Tears/Skin Trauma/Skin Stripping/Skin Flap/Skin Ulcer/Friction injury/Epidermal Stripping/Epidermal Strippings/Skin Ulcers. Também se utilizou os termos do Medical Subject Headings Term (MeSH) para busca na base MEDLINE, e Descritores em Ciência da Saúde (DECs) na base LILACS. Para delimitar a estratégia de busca aplicaram-se os operadores booleanos AND e OR. As palavras-chave foram pesquisadas em português, espanhol e inglês. A busca nas bases de dados foi realizada em maio de 2021.

Foram definidos como critérios de inclusão: artigos de pesquisas que apresentassem em seu título ou no resumo a temática relacionada com seres humanos na faixa etária igual ou maior a 60 anos, disponíveis nas línguas inglesa, portuguesa ou espanhola, publicados entre os anos de 2014 e 2020. Foram excluídas produções científicas duplicadas, editoriais, cartas, relatos de experiência, análise secundária, comentários e resumo de 
anais; livros, dissertações, teses, programas e políticas de saúde.

Ao término da seleção dos estudos, os artigos foram armazenados e sintetizados em ordem sequencial em documento Software Microsoft Word ${ }^{\circledR}$ por um dos pesquisadores. Os resultados foram apresentados em quadro contendo o nível de evidência (NE), ano, título, autor, país, método e base de dados dos artigos analisados, e o corpus de análise resultou em quatro categorias temáticas.

\section{Resultados}

Foram identificados 107.295 estudos, resultando em 42 publicações apontadas elegíveis para análise. Para tal, as etapas de cada processo em consonância ao modelo PRISMA (2009) estão representadas na Figura 1.

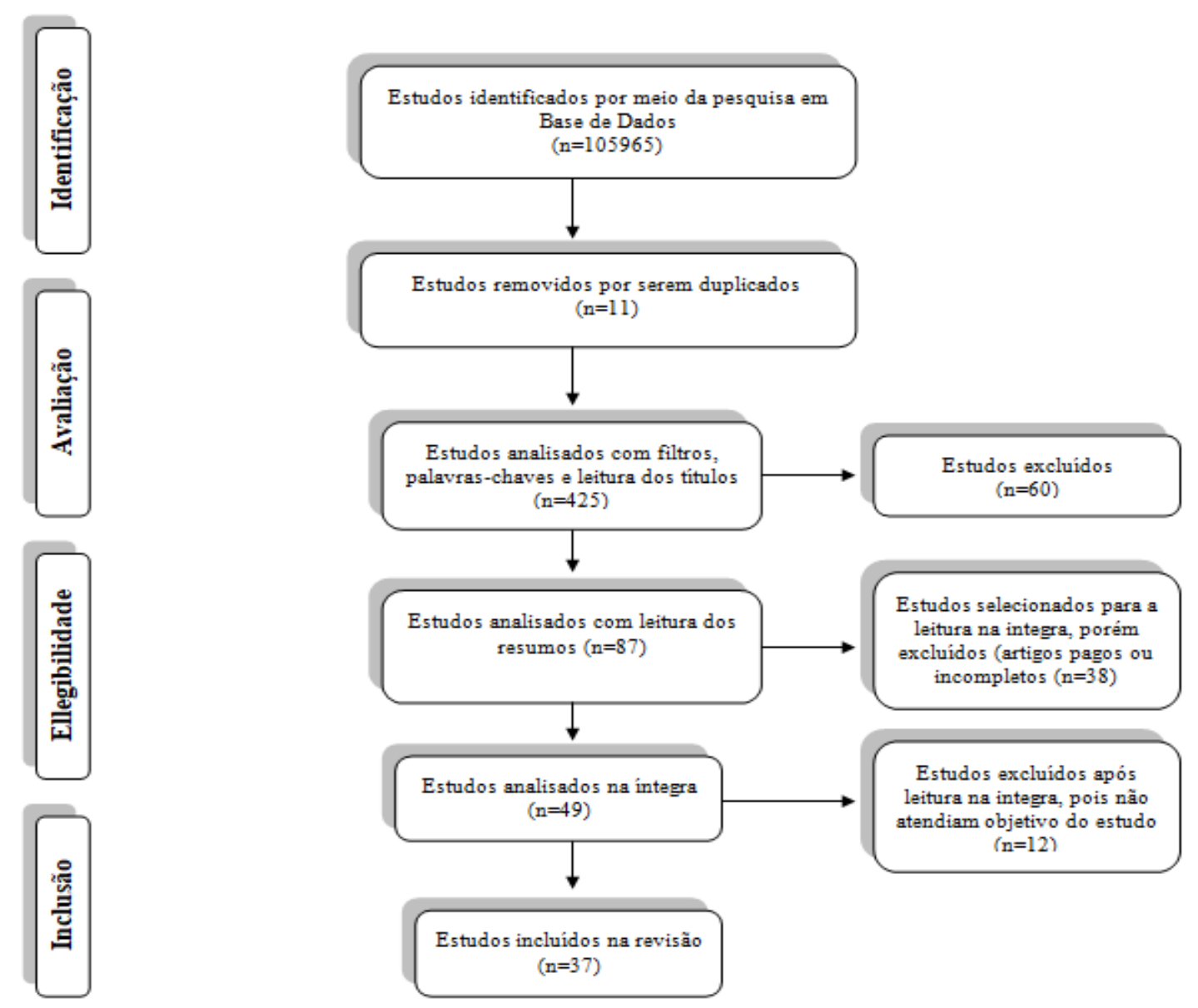

Figura 1 - Fluxograma do processo de inclusão e exclusão dos estudos.

Em seguida a definição dos estudos incluídos para análise, as publicações que compuseram as amostras foram organizadas e demonstradas por meio do Quadro 1. 
Quadro 1 - Estudos incluídos na revisão integrativa quanto ao ano, nível de evidência autoria, título da publicação, objetivo, país, método, base dados e nível de evidência

\begin{tabular}{|c|c|c|c|c|c|}
\hline $\begin{array}{l}\text { Ano } \\
\text { NE* }\end{array}$ & Autoria e Título & Objetivo & País & Método & Base \\
\hline $\begin{array}{c}2020 \\
\text { NE:VI }\end{array}$ & $\begin{array}{c}\text { Soares et al. (4) } \\
\text { Temperatura da } \\
\text { pele de diferentes } \\
\text { áreas corporais de } \\
\text { idosos sem risco } \\
\text { para lesões por } \\
\text { pressão. }\end{array}$ & $\begin{array}{l}\text { Identificar a temperatura da pele } \\
\text { de diferentes áreas corporais de } \\
\text { idosos hospitalizados em unidade } \\
\text { de clínica cirúrgica sem risco de } \\
\text { desenvolver lesões por pressão a } \\
\text { partir da Escala de Braden. }\end{array}$ & Brasil & $\begin{array}{c}\text { Transversal } \\
\text { descritivo }\end{array}$ & LILACS \\
\hline $\begin{array}{c}2020 \\
\text { NE:IV }\end{array}$ & $\begin{array}{l}\text { Moffat et al. (5) } \\
\text { Impact of a patient- } \\
\text { specific national } \\
\text { programme aimed } \\
\text { at increasing the use } \\
\text { of emollient } \\
\text { moisturisers to } \\
\text { reduce the risk of } \\
\text { skin tears: a } \\
\text { longitudinal cohort } \\
\text { study. } \\
\end{array}$ & $\begin{array}{c}\text { Avaliar o impacto de um } \\
\text { programa nacional específico } \\
\text { para pacientes, voltado para } \\
\text { idosos australianos e profissionais } \\
\text { de saúde, com o objetivo de } \\
\text { aumentar o uso de hidratantes } \\
\text { emolientes para reduzir o risco de } \\
\text { Skin tears. }\end{array}$ & Austrália & $\begin{array}{c}\text { Coorte } \\
\text { retrospectiva }\end{array}$ & PUBMED \\
\hline $\begin{array}{c}2019 \\
\text { NE:VI }\end{array}$ & $\begin{array}{l}\text { Yap et al. (6) } \\
\text { Pressure Injury } \\
\text { Prevention: } \\
\text { Outcomes and } \\
\text { Challenges to Use } \\
\text { of Resident } \\
\text { Monitoring } \\
\text { Technology in a } \\
\text { Nursing Home. }\end{array}$ & $\begin{array}{l}\text { Examinar a usabilidade, as } \\
\text { percepções do usuário e a } \\
\text { subcultura ocupacional da } \\
\text { enfermagem associada à } \\
\text { introdução de um sistema de } \\
\text { monitoramento do paciente para } \\
\text { facilitar a implementação do } \\
\text { tratamento padrão pela equipe de } \\
\text { enfermagem para a prevenção de } \\
\text { úlceras por pressão / lesões no } \\
\text { ambiente do lar de idosos. }\end{array}$ & $\begin{array}{c}\text { Estados Unidos } \\
\text { da América }\end{array}$ & Pesquisa Mista & PUBMED \\
\hline $\begin{array}{c}2019 \\
\text { NE:VII }\end{array}$ & $\begin{array}{l}\text { Lavallée et al. (7) } \\
\text { Preventing pressure } \\
\text { injury in nursing } \\
\text { homes: developing } \\
\text { a care bundle using } \\
\text { the Behaviour } \\
\text { Change Wheel. }\end{array}$ & $\begin{array}{c}\text { Desenvolver, com enfermeiros } \\
\text { especialistas e equipe de } \\
\text { enfermagem em casa de saúde, } \\
\text { um pacote de cuidados } \\
\text { de prevenção de lesões por } \\
\text { pressão baseado em teoria e } \\
\text { evidências para uso em ambientes } \\
\text { de casa de saúde. }\end{array}$ & Inglaterra & $\begin{array}{l}\text { Quantitativo } \\
\text { Metodológico }\end{array}$ & PUBMED \\
\hline $\begin{array}{c}2019 \\
\text { NE:IV }\end{array}$ & $\begin{array}{l}\text { Eglseer et al. (8) } \\
\text { Nutritional } \\
\text { management of } \\
\text { older hospitalised } \\
\text { patients with } \\
\text { pressure injuries. }\end{array}$ & $\begin{array}{c}\text { Descrever as intervenções } \\
\text { nutricionais realizadas em } \\
\text { pacientes austríacos } \\
\text { hopitalizados, com } 70 \text { anos de } \\
\text { idade ou mais e com (risco de } \\
\text { desenvolver) lesões por pressão. }\end{array}$ & Austrália & $\begin{array}{c}\text { Transversal } \\
\text { multicêntrico. }\end{array}$ & PUBMED \\
\hline $\begin{array}{c}2018 \\
\text { NE:IV }\end{array}$ & $\begin{array}{l}\text { Rondinelli et al. (9) } \\
\text { Hospital-Acquired } \\
\text { Pressure Injury: } \\
\text { Risk-Adjusted } \\
\text { Comparisons in an } \\
\text { Integrated } \\
\text { Healthcare Delivery } \\
\text { system. } \\
\end{array}$ & $\begin{array}{l}\text { Descrever a incidência de lesões } \\
\text { por pressão adquiridas em } \\
\text { hospitais, os fatores de risco e a } \\
\text { variação hospitalar ajustada ao } \\
\text { risco em um sistema integrado de } \\
\text { saúde da Califórnia. }\end{array}$ & $\begin{array}{l}\text { Estados } \\
\text { Unidos da } \\
\text { América }\end{array}$ & $\begin{array}{c}\text { Coorte } \\
\text { retrospectiva }\end{array}$ & PUBMED \\
\hline $\begin{array}{c}2018 \\
\text { NE:VI }\end{array}$ & $\begin{array}{l}\text { Gray et al. (10) } \\
\text { Opportunities for } \\
\text { better value wound } \\
\text { care: a multiservice, } \\
\text { cross-sectional } \\
\text { survey of complex } \\
\text { wounds and their } \\
\text { care in a UK } \\
\text { community } \\
\text { population. }\end{array}$ & $\begin{array}{l}\text { Quantificar o número, tipo e } \\
\text { gestão de feridas complexas } \\
\text { tratadas ao longo de um período } \\
\text { de duas semanas e explorar as } \\
\text { variações nos cuidados, } \\
\text { comparando as práticas atuais de } \\
\text { avaliação, prevenção e tratamento } \\
\text { de feridas }\end{array}$ & Inglaterra & $\begin{array}{c}\text { Transversal } \\
\text { descritivo }\end{array}$ & PUBMED \\
\hline
\end{tabular}


Vittalle - Revista de Ciências da Saúde v. 33, n. 3 (2021) 96-111

\begin{tabular}{|c|c|c|c|c|c|}
\hline $\begin{array}{c}2018 \\
\text { NE:IV }\end{array}$ & $\begin{array}{c}\text { Alderden (11) } \\
\text { Outcomes } \\
\text { associated with } \\
\text { stage } 1 \text { pressure } \\
\text { injuries: a } \\
\text { retrospective cohort } \\
\text { study. }\end{array}$ & $\begin{array}{c}\text { Examinar os resultados das lesões } \\
\text { por pressão em estágio } 1 \text { entre } \\
\text { pacientes em terapia intensiva e } \\
\text { identificar os fatores associados } \\
\text { ao agravamento das lesões por } \\
\text { pressão. }\end{array}$ & $\begin{array}{l}\text { Estados } \\
\text { Unidos da } \\
\text { América }\end{array}$ & $\begin{array}{c}\text { Coorte } \\
\text { retrospectiva }\end{array}$ & CINAHL \\
\hline $\begin{array}{c}2018 \\
\text { NE:VI }\end{array}$ & $\begin{array}{l}\text { Kaşikçi et al. (12) } \\
\text { Investigation of the } \\
\text { prevalence of } \\
\text { pressure ulcers and } \\
\text { patient-related risk } \\
\text { factors in hospitals } \\
\text { in the province of } \\
\text { Erzurum: A cross- } \\
\text { sectional study. }\end{array}$ & $\begin{array}{l}\text { Determinar a prevalência de } \\
\text { úlceras de pressão e fatores de } \\
\text { risco relacionados ao paciente em } \\
\text { pacientes internados em } \\
\text { tratamento na província de } \\
\text { Erzurum, localizada na parte } \\
\text { oriental da Turquia. }\end{array}$ & Turquia & $\begin{array}{l}\text { Quantitativo } \\
\text { Descritivo }\end{array}$ & PUBMED \\
\hline $\begin{array}{c}2018 \\
\text { NE:IV }\end{array}$ & $\begin{array}{l}\text { Strazzieri-Pulido et } \\
\text { al. (13) } \\
\text { Pressure injuries in } \\
\text { critical patients: } \\
\text { incidence patient- } \\
\text { associated factores } \\
\text { and nursing } \\
\text { workload }\end{array}$ & $\begin{array}{l}\text { Estimar a incidência de lesão por } \\
\text { pressão e seus preditores, } \\
\text { incluindo a carga de trabalho de } \\
\text { enfermagem em pacientes } \\
\text { críticos. }\end{array}$ & Brasil & $\begin{array}{c}\text { Coorte } \\
\text { retrospectiva }\end{array}$ & PUBMED \\
\hline $\begin{array}{c}2018 \\
\text { NE:VI }\end{array}$ & $\begin{array}{l}\text { Bermark et al. (14) } \\
\text { Prevalence of skin } \\
\text { tears in the } \\
\text { extremities in } \\
\text { inpatients at a } \\
\text { hospital in } \\
\text { Denmark. }\end{array}$ & $\begin{array}{l}\text { Determinar a prevalência de skin } \\
\text { teares nas extremidades e } \\
\text { explorar fatores associados ao } \\
\text { desenvolvimento de skin tear em } \\
\text { pacientes internados em um } \\
\text { hospital dinamarquês. }\end{array}$ & Dinamarca & $\begin{array}{c}\text { Quantitativo } \\
\text { Descritivo }\end{array}$ & PUBMED \\
\hline $\begin{array}{c}2018 \\
\text { NE:VI }\end{array}$ & $\begin{array}{l}\text { Han et al. (15) } \\
\text { Predictors of } \\
\text { hospital-acquired } \\
\text { pressure ulcers } \\
\text { among older adult } \\
\text { inpatients. }\end{array}$ & $\begin{array}{l}\text { Fornecer informações básicas } \\
\text { sobre os cuidados preventivos de } \\
\text { úlcera por pressão, por meio da } \\
\text { análise das características } \\
\text { relacionadas à úlcera por pressão } \\
\text { e da identificação de preditores } \\
\text { de úlcera por pressão. }\end{array}$ & $\begin{array}{l}\text { Estados } \\
\text { Unidos da } \\
\text { América }\end{array}$ & $\begin{array}{c}\text { Quantitativo } \\
\text { Descritivo }\end{array}$ & PUBMED \\
\hline $\begin{array}{c}2018 \\
\text { NE:VI }\end{array}$ & $\begin{array}{l}\text { Kim et al. (16) } \\
\text { The relation ship of } \\
\text { subepidermal } \\
\text { moisture and early } \\
\text { stage pressure } \\
\text { injury by visual skin } \\
\text { assessment. }\end{array}$ & $\begin{array}{l}\text { Examinar a relação entre a } \\
\text { umidade subepidérmica e a lesão } \\
\text { por pressão em estágio inicial por } \\
\text { avaliação visual da pele em } \\
\text { idosos coreanos. }\end{array}$ & $\begin{array}{l}\text { Coréia do } \\
\text { Sul }\end{array}$ & $\begin{array}{c}\text { Observacional } 1 \\
\text { ongitudinal }\end{array}$ & PUBMED \\
\hline $\begin{array}{c}2018 \\
\text { NE:IV }\end{array}$ & $\begin{array}{c}\text { Sardo (17) } \\
\text { Pressure ulcer } \\
\text { incidence and } \\
\text { braden subscales: } \\
\text { retrospective cohort } \\
\text { analysis in general } \\
\text { wards of portuguese } \\
\text { hospital. } \\
\end{array}$ & $\begin{array}{l}\text { Estudar a influência dos escores } \\
\text { das subescalas de Braden (na } \\
\text { primeira avaliação de risco de } \\
\text { úlcera por pressão) na incidência } \\
\text { de úlcera por pressão usando uma } \\
\text { análise univariada e uma } \\
\text { multivariada de tempo para o } \\
\text { evento. }\end{array}$ & Portugal & $\begin{array}{c}\text { Coorte } \\
\text { prospectivo }\end{array}$ & PUBMED \\
\hline $\begin{array}{c}2018 \\
\text { NE:VI }\end{array}$ & $\begin{array}{l}\text { Karahan et al. (18) } \\
\\
\text { Factors Affecting } \\
\text { Wound Healing in } \\
\text { Individuals With } \\
\text { Pressure Ulcers: A } \\
\text { Retrospective } \\
\text { Study. }\end{array}$ & $\begin{array}{l}\text { Identificar os fatores que podem } \\
\text { afetar a cura de úlcera por pressão } \\
\text { em pacientes adultos. }\end{array}$ & Turquia & $\begin{array}{c}\text { Coorte } \\
\text { retrospectivo }\end{array}$ & PUBMED \\
\hline
\end{tabular}




\begin{tabular}{|c|c|c|c|c|c|}
\hline $\begin{array}{c}2018 \\
\text { NE:VI }\end{array}$ & $\begin{array}{l}\text { Chiang et al. (19) } \\
\text { Surgical treatment } \\
\text { and strategy in } \\
\text { patients with } \\
\text { multiple pressure } \\
\text { sores. }\end{array}$ & $\begin{array}{l}\text { Apresentar tratamento cirúrgico e } \\
\text { estratégia para pacientes com } \\
\text { múltiplas úlceras de pressão. }\end{array}$ & China & Estudo de caso & PUBMED \\
\hline $\begin{array}{l}2018 \\
\text { NE:II }\end{array}$ & $\begin{array}{l}\text { Tarajad et al. (20) } \\
\text { Effect of laser } \\
\text { therapy on } \\
\text { expression of angio } \\
\text { and fibrogenic } \\
\text { factors, and } \\
\text { cytokine } \\
\text { concentrations } \\
\text { during the healing } \\
\text { process of human } \\
\text { pressure ulcers }\end{array}$ & $\begin{array}{l}\text { Avaliar o efeito da irradiação } \\
\text { laser em diferentes comprimentos } \\
\text { de onda na expressão de fatores } \\
\text { de crescimento selecionados e } \\
\text { mediadores inflamatórios em } \\
\text { estágios específicos do processo } \\
\text { de cicatrização de feridas. }\end{array}$ & Austrália & $\begin{array}{l}\text { Ensaio Clínico } \\
\text { Randomizado }\end{array}$ & PUBMED \\
\hline $\begin{array}{c}2017 \\
\text { NE:IV }\end{array}$ & $\begin{array}{l}\text { Smith et al. (21) } \\
\text { Exploring the role } \\
\text { of pain as an early } \\
\text { predictor of } \\
\text { category } 2 \text { pressure } \\
\text { ulcers: a } \\
\text { prospective cohort } \\
\text { study }\end{array}$ & $\begin{array}{l}\text { Explorar a dor relacionada à área } \\
\text { de pressão como um preditor do } \\
\text { desenvolvimento de úlcera de } \\
\text { pressão (UP) de categoria } \geq 2 .\end{array}$ & Ucrânia & $\begin{array}{c}\text { Coorte } \\
\text { prospectivo } \\
\text { multicêntrico. }\end{array}$ & PUBMED \\
\hline $\begin{array}{c}2017 \\
\text { NE:VI }\end{array}$ & $\begin{array}{l}\text { Skogestd et al. (22) } \\
\text { Supplementing the } \\
\text { Braden scale for } \\
\text { pressure ulcer risk } \\
\text { among medical } \\
\text { inpatients: the } \\
\text { contribution of self- } \\
\text { reported symptoms } \\
\text { and standard } \\
\text { laboratory tests } \\
\end{array}$ & $\begin{array}{c}\text { Avaliar a experiência dos } \\
\text { sintomas de pacientes } \\
\text { hospitalizados e os resultados de } \\
\text { sangue de laboratório } \\
\text { selecionados como indicadores de } \\
\text { seu risco de úlcera por pressão, } \\
\text { medido pela escala de Braden. }\end{array}$ & Noruega & $\begin{array}{l}\text { Transversal. } \\
\text { Observacional } \\
\text { descritivo } \\
\text { (prospectivo). }\end{array}$ & PUBMED \\
\hline $\begin{array}{l}2017 \\
\text { NE:II }\end{array}$ & $\begin{array}{l}\text { Walker et al. (23) } \\
\text { A Pilot Randomized } \\
\text { Controlled Trial } \\
\text { Using Prophylactic } \\
\text { Dressings to } \\
\text { Minimize Sacral } \\
\text { Pressure Injuries in } \\
\text { High-Risk } \\
\text { Hospitalized } \\
\text { Patients } \\
\end{array}$ & $\begin{array}{l}\text { Examinar o efeito de curativos } \\
\text { profiláticos para minimizar as } \\
\text { lesões por pressão sacral em } \\
\text { pacientes hospitalizados de alto } \\
\text { risco e avaliar os critérios de } \\
\text { viabilidade do estudo. }\end{array}$ & Austrália & $\begin{array}{l}\text { Ensaio Clínico } \\
\text { Randomizado }\end{array}$ & PUBMED \\
\hline $\begin{array}{c}2016 \\
\text { NE:VI }\end{array}$ & $\begin{array}{c}\text { Seval et al. (24) } \\
\text { A Prospective, } \\
\text { Descriptive Study } \\
\text { to Determine the } \\
\text { Rate and } \\
\text { Characteristics of } \\
\text { and Risk Factors for } \\
\text { the Development of } \\
\text { Medical Device- } \\
\text { related Pressure } \\
\text { Ulcers in Intensive } \\
\text { Care Units. }\end{array}$ & $\begin{array}{l}\text { Determinar a prevalência, os } \\
\text { fatores de risco e as } \\
\text { características de úlceras por } \\
\text { pressão adquiridas em hospitais } \\
\text { relacionadas a dispositivos } \\
\text { médicos }\end{array}$ & Turquia & $\begin{array}{l}\text { Transversal. } \\
\text { Observacional } \\
\text { descritivo. }\end{array}$ & PUBMED \\
\hline $\begin{array}{c}2016 \\
\text { NE:VI }\end{array}$ & $\begin{array}{l}\text { Lannerin et al. (25) } \\
\text { Factors related to } \\
\text { falls, weight-loss } \\
\text { and pressure ulcers- } \\
\text {-more insight in risk } \\
\text { assessment among } \\
\text { nursing home } \\
\text { residents }\end{array}$ & $\begin{array}{c}\text { Descrever como os itens } \\
\text { incluídos em três escalas } \\
\text { diferentes, Índice de Risco de } \\
\text { Queda de Downton, a forma } \\
\text { abreviada de Mini Avaliação } \\
\text { Nutricional e a Escala de Norton } \\
\text { Modificada estão associados a } \\
\text { desfechos graves como quedas, } \\
\text { perda de peso e úlceras por } \\
\text { pressão }\end{array}$ & Suécia & $\begin{array}{c}\text { Coorte } \\
\text { Longitudinal } \\
\text { descritivo. }\end{array}$ & PUBMED \\
\hline
\end{tabular}




\begin{tabular}{|c|c|c|c|c|c|}
\hline $\begin{array}{c}2016 \\
\text { NE:VI }\end{array}$ & $\begin{array}{l}\text { Mizokami et al. } \\
\text { (26) } \\
\text { Pressure ulcers } \\
\text { induced by drug } \\
\text { administration: A } \\
\text { new concept and } \\
\text { report of four cases } \\
\text { in elderly patients } \\
\end{array}$ & $\begin{array}{l}\text { Propor que as úlceras por pressão } \\
\text { induzidas por medicamento são } \\
\text { úlceras por pressão causadas por } \\
\text { uma força externa que ocorre } \\
\text { após a administração do } \\
\text { medicamento. }\end{array}$ & Japão & Estudo de Caso. & PUBMED \\
\hline $\begin{array}{l}2016 \\
\text { NE:IV }\end{array}$ & $\begin{array}{l}\text { Setoguch et al. (27) } \\
\text { Predictability of } \\
\text { Pressure Ulcers } \\
\text { Based on Operation } \\
\text { Duration, Transfer } \\
\text { Activity, and Body } \\
\text { Mass Index } \\
\text { Through the Use of } \\
\text { an Alternating } \\
\text { Decision Tree } \\
\end{array}$ & $\begin{array}{l}\text { Desenvolver um modelo de } \\
\text { previsão para os casos de úlcera } \\
\text { por pressão que continuam a } \\
\text { ocorrer em um hospital de } \\
\text { cuidados intensivos com uma } \\
\text { baixa taxa de ocorrência de } \\
\text { úlceras por pressão. }\end{array}$ & Japão & Caso-Controle. & PUBMED \\
\hline $\begin{array}{l}2016 \\
\text { NE:VI }\end{array}$ & $\begin{array}{l}\text { Park e Choi (28) } \\
\text { Prospective study } \\
\text { on Incontinence- } \\
\text { Associated } \\
\text { Dermatitis and its } \\
\text { Severity instrument } \\
\text { for verifying its } \\
\text { ability to predict the } \\
\text { development of } \\
\text { pressure ulcers in } \\
\text { patients with fecal } \\
\text { incontinence } \\
\end{array}$ & $\begin{array}{l}\text { Determinar a viabilidade de } \\
\text { aplicação do instrumento } \\
\text { Dermatite Associada à } \\
\text { Incontinência e sua Gravidade } \\
\text { (em pacientes com incontinência } \\
\text { fecal como uma ferramenta para } \\
\text { prever o desenvolvimento de } \\
\text { úlcera de pressão }\end{array}$ & $\begin{array}{l}\text { Coréia do } \\
\text { Sul }\end{array}$ & $\begin{array}{c}\text { Coorte } \\
\text { Prospectivo } \\
\text { descritivo. }\end{array}$ & PUBMED \\
\hline $\begin{array}{l}2016 \\
\text { NE:VI }\end{array}$ & $\begin{array}{c}\text { Kang,Tzeng e } \\
\text { Miller(29) } \\
\text { Facility } \\
\text { Characteristics and } \\
\text { Risk of Developing } \\
\text { Pressure Ulcers in } \\
\text { US Nursing Homes. }\end{array}$ & $\begin{array}{c}\text { Prever probabilidades ajustadas } \\
\text { ao risco de úlceras de pressão } \\
\text { com } 4 \text { estágios utilizando um } \\
\text { modelo de regressão logística } \\
\text { multinomial. }\end{array}$ & $\begin{array}{l}\text { Estados } \\
\text { Unidos da } \\
\text { América }\end{array}$ & $\begin{array}{l}\text { Quantitativo } \\
\text { descritivo. }\end{array}$ & PUBMED \\
\hline $\begin{array}{l}2016 \\
\text { NE:VI }\end{array}$ & $\begin{array}{l}\text { Athlin et al. (30) } \\
\text { Heel pressure ulcer, } \\
\text { prevention and } \\
\text { predictors during } \\
\text { the care delivery } \\
\text { chain - when and } \\
\text { where to take } \\
\text { action? A } \\
\text { descriptive and } \\
\text { explorative study. }\end{array}$ & $\begin{array}{l}\text { Descrever a prevalência de úlcera } \\
\text { por pressão no calcanhar e as } \\
\text { ações de enfermagem em relação } \\
\text { à prevenção de úlcera por pressão } \\
\text { na cadeia de atendimento para } \\
\text { pacientes mais velhos com } \\
\text { sintomas neurológicos ou } \\
\text { condição geral reduzida, bem } \\
\text { como investigar os preditores } \\
\text { precoces para o desenvolvimento } \\
\text { de úlcera de pressão no calcanhar } \\
\text { durante a cadeia de atendimento. }\end{array}$ & Suécia & $\begin{array}{l}\text { Prospectivo, } \\
\text { exploratório } \\
\text { descritivo. }\end{array}$ & PUBMED \\
\hline $\begin{array}{l}2016 \\
\text { NE:VI }\end{array}$ & $\begin{array}{l}\text { Kwong, Hung e } \\
\text { Woo (31) } \\
\text { Improvement of } \\
\text { pressure ulcer } \\
\text { prevention care in } \\
\text { private for-profit } \\
\text { residential care } \\
\text { homes: an action } \\
\text { research study. } \\
\end{array}$ & $\begin{array}{l}\text { Desenvolver um protocolo de } \\
\text { prevenção de úlcera de pressão } \\
\text { para lares de idosos privados com } \\
\text { fins lucrativos. }\end{array}$ & China & Pesquisa-ação. & PUBMED \\
\hline $\begin{array}{l}2016 \\
\text { NE:IV }\end{array}$ & $\begin{array}{l}\text { Baath et al. (32) } \\
\text { Prevention of heel } \\
\text { pressure ulcers } \\
\text { among older } \\
\text { patients from } \\
\text { ambulance care to } \\
\text { hospital discharge: } \\
\text { A multi-centre }\end{array}$ & $\begin{array}{c}\text { Investigar o efeito de uma } \\
\text { intervenção precoce, uma bota } \\
\text { com dispositivo de suspensão de } \\
\text { calcanhar, na incidência de } \\
\text { úlceras de pressão de calcanhar } \\
\text { em pacientes mais velhos (com } \\
\text { mais de } 70 \text { anos). }\end{array}$ & Suécia & Caso-controle. & PUBMED \\
\hline
\end{tabular}




\begin{tabular}{|c|c|c|c|c|c|}
\hline & $\begin{array}{l}\text { randomized } \\
\text { controlled trial. }\end{array}$ & & & & \\
\hline $\begin{array}{l}2016 \\
\text { NE:VI }\end{array}$ & $\begin{array}{l}\text { Baranoski et al (33) } \\
\text { Preventing, } \\
\text { Assessing, and } \\
\text { Managing Skin } \\
\text { Tears: A Clinical } \\
\text { Review. }\end{array}$ & $\begin{array}{l}\text { Descrever as complicações que se } \\
\text { desenvolveram em um paciente } \\
\text { idoso cuja lesão por fricção foi } \\
\text { coberta inadequadamente e } \\
\text { discutir as melhores práticas para } \\
\text { prevenir, avaliar, documentar e } \\
\text { controlar as lesões por fricção. }\end{array}$ & Não especif. & Estudo de caso. & PUBMED \\
\hline $\begin{array}{l}2016 \\
\text { NE:IV }\end{array}$ & $\begin{array}{l}\text { Niu et al. (34) } \\
\text { Therapeutic Effect } \\
\text { of External } \\
\text { Application of } \\
\text { Ligustrazine } \\
\text { Combined with } \\
\text { Holistic Nursing on } \\
\text { Pressure Sores. } \\
\end{array}$ & $\begin{array}{l}\text { Explorar o efeito terapêutico da } \\
\text { aplicação externa de ligustrazina } \\
\text { combinada com enfermagem } \\
\text { holística em úlceras de pressão, } \\
\text { bem como o mecanismo } \\
\text { subjacente. }\end{array}$ & China & Caso-Controle & PUBMED \\
\hline $\begin{array}{c}2016 \\
\text { NE:VI }\end{array}$ & $\begin{array}{l}\text { Zeigler et al. (35) } \\
\\
\text { Use of the Pressure } \\
\text { Ulcer Scale for } \\
\text { Healing (PUSH) in } \\
\text { Inpatient } \\
\text { Rehabilitation: A } \\
\text { Case Example. }\end{array}$ & $\begin{array}{l}\text { Descrever o uso da Escala de } \\
\text { Úlcera de Pressão para Cura } \\
\text { (PUSH), que demonstrou } \\
\text { excelentes propriedades } \\
\text { psicométricas, para avaliar, } \\
\text { monitorar o progresso e orientar a } \\
\text { tomada de decisão clínica durante } \\
\text { a reabilitação hospitalar. }\end{array}$ & $\begin{array}{l}\text { Estados } \\
\text { Unidos da } \\
\text { América }\end{array}$ & Estudo de Caso & PUBMED \\
\hline $\begin{array}{c}2016 \\
\text { NE:VII }\end{array}$ & $\begin{array}{l}\text { Santos et al. (36) } \\
\text { Diagnóstico de } \\
\text { enfermagem risco } \\
\text { de úlcera por } \\
\text { pressão: validação } \\
\text { de conteúdo. } \\
\end{array}$ & $\begin{array}{l}\text { Validar o conteúdo do novo } \\
\text { diagnóstico de enfermagem, } \\
\text { denominado risco de úlcera por } \\
\text { pressão. }\end{array}$ & Brasil & $\begin{array}{l}\text { Validação de } \\
\text { conteúdo, } \\
\text { metodológico }\end{array}$ & SCIELO \\
\hline $\begin{array}{l}2015 \\
\text { NE:VI }\end{array}$ & $\begin{array}{l}\text { Duim et al. (37) } \\
\text { Prevalência e } \\
\text { características das } \\
\text { feridas em pessoas } \\
\text { idosas residentes na } \\
\text { comunidade. } \\
\end{array}$ & $\begin{array}{l}\text { Descrever o perfil e as } \\
\text { características de idosos com } \\
\text { restrição de mobilidade, } \\
\text { residentes na comunidade e com } \\
\text { presença de lesões de pele. }\end{array}$ & Brasil & $\begin{array}{l}\text { Quantitativo. } \\
\text { Exploratório e } \\
\text { descritivo. }\end{array}$ & LILACS \\
\hline $\begin{array}{l}2015 \\
\text { NE:VI }\end{array}$ & $\begin{array}{l}\text { Rasero et al. (38) } \\
\text { Pressure Ulcers in } \\
\text { Older Adults: A } \\
\text { Prevalence Study. }\end{array}$ & $\begin{array}{l}\text { Medir a prevalência de úlcera por } \\
\text { pressão em uma população idosa. }\end{array}$ & Itália & $\begin{array}{l}\text { Transversal. } \\
\text { Observacional } \\
\text { descritivo. }\end{array}$ & PUBMED \\
\hline $\begin{array}{l}2015 \\
\text { NE:IV }\end{array}$ & $\begin{array}{l}\text { Lewin et al. (39) } \\
\text { Identification of } \\
\text { risk factors } \\
\text { associated with the } \\
\text { development of skin } \\
\text { tears in hospitalised } \\
\text { older persons: a } \\
\text { case-control study. }\end{array}$ & $\begin{array}{c}\text { Identificar os fatores de risco } \\
\text { associados ao desenvolvimento } \\
\text { de lesões cutâneas em pessoas } \\
\text { idosas }\end{array}$ & Austrália & Caso-Controle. & PUBMED \\
\hline $\begin{array}{l}2015 \\
\text { NE:IV }\end{array}$ & $\begin{array}{l}\text { Sardo et al. (40) } \\
\text { Pressure ulcer risk } \\
\text { assessment: } \\
\text { retrospective } \\
\text { analysis of Braden } \\
\text { Scale scores in } \\
\text { Portuguese } \\
\text { hospitalised adult } \\
\text { patients. }\end{array}$ & $\begin{array}{l}\text { Analisar os escores e subescores } \\
\text { da escala de Braden avaliados em } \\
\text { pacientes portugueses adultos } \\
\text { hospitalizados em associação } \\
\text { com as suas características, } \\
\text { diagnósticos e tempo de } \\
\text { internamento. }\end{array}$ & Portugal & $\begin{array}{c}\text { Coorte } \\
\text { prospectivo }\end{array}$ & PUBMED \\
\hline
\end{tabular}




\begin{tabular}{|c|c|c|c|c|c|}
\hline $\begin{array}{l}2015 \\
\text { NE:IV }\end{array}$ & $\begin{array}{l}\text { Sanada et al. (41) } \\
\text { Incidence of skin } \\
\text { tears in the } \\
\text { extremities among } \\
\text { elderly patients at a } \\
\text { long-term medical } \\
\text { facility in Japan: A } \\
\text { prospective cohort } \\
\text { study. }\end{array}$ & $\begin{array}{c}\text { Estimar a incidência cumulativa } \\
\text { de lesão por fricção e identificar } \\
\text { seu fator de risco. }\end{array}$ & Japão & $\begin{array}{c}\text { Coorte } \\
\text { prospectivo }\end{array}$ & PUBMED \\
\hline $\begin{array}{c}2015 \\
\text { NE:VI }\end{array}$ & $\begin{array}{l}\text { Dijkstra et al. (42) } \\
\text { Using the Care } \\
\text { Dependency Scale } \\
\text { for identifying } \\
\text { patients at risk for } \\
\text { pressure ulcer. }\end{array}$ & $\begin{array}{c}\text { Avaliar a triagem de risco para } \\
\text { úlcera de pressão usando a Care } \\
\text { Dependency Scale (CDS) para } \\
\text { pacientes que recebem cuidados } \\
\text { domiciliares ou internados em } \\
\text { uma residência ou lar de idosos } \\
\text { na Holanda. } \\
\end{array}$ & Holanda & $\begin{array}{l}\text { Quantitativo } \\
\text { descritivo. }\end{array}$ & PUBMED \\
\hline $\begin{array}{c}2015 \\
\text { NE:VI }\end{array}$ & $\begin{array}{c}\text { Jaul et al. (43) } \\
\text { A comparative, } \\
\text { descriptive study of } \\
\text { systemic factors and } \\
\text { survival in elderly } \\
\text { patients with sacral } \\
\text { pressure ulcers. } \\
\end{array}$ & $\begin{array}{l}\text { Identificar os fatores sistêmicos } \\
\text { do paciente associados às úlceras } \\
\text { de pressão sacrais e para } \\
\text { comparar o tempo de sobrevida } \\
\text { em pacientes com e sem úlceras } \\
\text { de pressão. }\end{array}$ & Israel & $\begin{array}{l}\text { Quantitativo } \\
\text { descritivo. }\end{array}$ & PUBMED \\
\hline $\begin{array}{l}2015 \\
\text { NE:VI }\end{array}$ & $\begin{array}{l}\text { Iizaka et al. (44) } \\
\text { Clinical validity of } \\
\text { the estimated } \\
\text { energy requirement } \\
\text { and the average } \\
\text { protein requirement } \\
\text { for nutritional status } \\
\text { change and wound } \\
\text { healing in older } \\
\text { patients with } \\
\text { pressure ulcers: A } \\
\text { multicenter } \\
\text { prospective cohort } \\
\text { study. }\end{array}$ & $\begin{array}{c}\text { Avaliar a validade clínica desses } \\
\text { requisitos em pacientes idosos } \\
\text { hospitalizados com úlceras de } \\
\text { pressão, avaliando o estado } \\
\text { nutricional e cicatrização de } \\
\text { feridas. }\end{array}$ & Japão & $\begin{array}{c}\text { Prospectivo } \\
\text { multicêntrico. }\end{array}$ & PUBMED \\
\hline $\begin{array}{c}2015 \\
\text { NE:VII }\end{array}$ & $\begin{array}{l}\text { Strazzieri-Pulido et } \\
\text { al. (45) } \\
\text { Adaptação cultural, } \\
\text { validade de } \\
\text { conteúdo e } \\
\text { confiabilidade inter } \\
\text { observadores do } \\
\text { "STAR Skin Tear } \\
\text { Classification } \\
\text { System". }\end{array}$ & $\begin{array}{l}\text { Realizar a adaptação cultural do } \\
\text { STAR Skin Tear Classification } \\
\text { System para a língua portuguesa } \\
\text { e testar a validade de conteúdo e } \\
\text { a confiabilidade } \\
\text { interexaminadores da versão } \\
\text { adaptada }\end{array}$ & Brasil & $\begin{array}{l}\text { Quantitativo } \\
\text { metodológico. }\end{array}$ & SCIELO \\
\hline
\end{tabular}

*NE: Nível de Evidência.

A base Pubmed, apresentou maior número de publicações (36); a origem delas na maior parte era dos Estados Unidos da América (7), seguida por Brasil e Austrália (5). A maior parte das publicações apresentou propensão para abordagem na linha de identificação de riscos para o desenvolvimento de LP e LF. Considerando o tipo de pesquisa, a mais frequente foi estudos de coorte (11). Em relação à qualidade dos artigos, dois foram classificados de acordo com o nível de evidência de Melnyk e Fineout-Overholt (46) e a maioria com nível de evidência fraca (36).

Para análise dos dados as publicações foram compiladas em quatro categorias temáticas apresentadas a seguir.

Categoria 1 - Identificando fatores de risco para o desenvolvimento de Lesões por Friç̧ão e Lesões por Pressão 
Nesta categoria são apresentados os estudos que apontam que se tratando da rapidez com que as LP e LF podem se desenvolver faz-se fundamental identificar os pacientes em risco e iniciar medidas preventivas seja no âmbito domiciliar quanto hospitalar. Sendo assim, tais medidas preventivas são possíveis a partir do momento em que identificamos tais fatores $(4,10-12-, 14,22,24-29,37-41,43,45)$.

Estudos identificaram a relação entre a idade avançada e o desenvolvimento de LP, principalmente quando se trata de idosos longevos ${ }^{22,29,43}$. Doravante, o aumento da idade é preditor no desenvolvimento destas nos mais variados cenários, principalmente em pacientes críticos $(12,22,37,45)$. Ainda, como resultado de síndromes geriátricas, como incontinência, mobilidade reduzida ou desnutrição, idosos em hospitais têm maior risco de desenvolver lesão por pressão.

Quanto à associação de LP e a cor da pele, não houve correlação, embora a maioria dos pacientes submetidos aos estudos fosse da cor da pele branca $(10,11)$.

Em relação às condições nutricionais, diversos estudos expressam a importância do reconhecimento da análise laboratorial, visto que, níveis baixos de albumina e hemograma apresentam-se como fatores de risco para LP $(11,12,22,37,43)$.

Estudos (12,37) mostram relação significativa entre a existência de LP e hipoalbuminemia, culminando com a redução da síntese de colágeno, refletindo na reparação tecidual.Há relação entre baixa concentração de hemoglobina e diminuição da oxigenação ( $\left.\mathrm{SpO}_{2}\right)$ e LP (11), assim como aumento da prevalência de anemia e menores níveis de hemoglobina e albumina ${ }^{43}$ nestes casos.

$\mathrm{O}$ estado nutricional evidenciado por alterações no índice de massa corporal (IMC) (baixo peso, sobrepeso ou obesidade) pode influenciar a ocorrência de LP $(11,22,27,43)$.

Outro fator de risco relevante é a mobilidade prejudicada (26); assim como o uso de medicamentos, como sedativos e anticoagulantes (25) e hipotensores (13) e, a incontinência urinária $(12,28)$ e fecal $(38)$.

Além desses aspectos já mencionados, estudo (4) nos alerta sobre o avanço do conhecimento acerca da temperatura da pele de idosos, com importantes implicações para a gestão do cuidado relacionado à prevenção de lesão por pressão nessa população. $O$ objetivo é que a mensuração da temperatura da pele facilite a tomada de decisão clínica do profissional no seu fazer cotidiano, uma vez que há evidências de que estas alterações sugerem o aparecimento de LP.

Em relação à Lesão por Fricção (LF), percebe-se um quantitativo menor de estudos, se comparado aos de LP, que abordem os fatores de risco de LF principalmente quando se referem às pesquisas na comunidade. Os fatores de risco identificados nas publicações foram: lesões cutâneas prévias,equimose, risco de quedas e escala de Braden com baixa pontuação $(14,39,41)$.

\section{Categoria 2 - Prevenção e manutenção da integridade cutânea}

Diante da complexidade do tema e da identificação de que as LP e LF são condições evitáveis na maioria das vezes, torna-se fundamental a implantação de um bom plano de avaliação de riscos para prevenir e gerenciar esse cuidado. Dessa forma é relevante a adoção de pacotes de cuidados implementando mudanças de

Comportamentos (7).

A maior parte estudos pertencentes a esta categoria, relacionam-se aos cuidados preventivos para as LP $(15,16,23,30-32)$, mas um deles apresenta o impacto de um programa nacional específico com o objetivo de aumentar o uso de hidratantes emolientes para reduzir o risco de LF em idosos (5).

Dentre os cuidados preventivos mais evidenciados na literatura, estão o reposicionamento e a inspeção frequente da pele (15). Nesse sentido recomenda-se: uso 
do dispositivo de suspensão do calcanhar (30-32), bem como superfícies de apoio, assento redutor de pressão em cadeiras de rodas e a suspensão do uso de absorventes para incontinência urinária (31). Outras intervenções incluem suplemento nutricional $(8,30,32)$, assim como, avaliação da medida relativa da água livre no tecido subepidérmico, realizada através de dispositivo médico16, e uso de espuma de silicone em proeminências ósseas (23). Um dos estudos apresenta um sistema de monitoramento do paciente para facilitar o reposicionamento em conformidade com os padrões de reposicionamento de idosos dependentes a cada 2 horas (6).

Categoria 3 - Diagnóstico de enfermagem da lesão por fricção e por pressão

Somente três estudos abordam essa temática, sendo dois deles com foco no diagnóstico de LP e somente um relacionado à LF $(17,36,45)$.

Estudo aponta que o DE "mobilidade prejudicada" e "atividade física limitada", devem estar presentes no gerenciamento do cuidado, independente do escore total da escala de Braden, pois ambos são preditivos do desenvolvimento de LP durante o percurso da internação (17).

Autores (36) possuem um papel de grande valia na história do diagnóstico de LP, visto que validaram e incorporaram à lista da International Nursing Diagnosis (NANDA) 2015 - 2017 o DE "Risco de Úlcera por pressão".

Em relação à LF, foi atestada a viabilidade do Skin Tear Classification System (STAR) permitindo o reconhecimento das características da lesão, elucidando ações para o êxito no cuidado de enfermagem (45).

\section{Categoria 4-Tratando as Lesões por Fricção e por Pressão}

As LP e LF apresentam-se como indicador importante da qualidade do atendimento, visto que muitos fatores de risco podem ser prevenidos ou minimizados, porém sabe-se da existência de fatores complexos que nem sempre são modificáveis, as quais levam às lesões de pele. Nessa categoria são elencados os estudos que versam sobre as possibilidades de tratamento para LP e LF (18-20,33-35,44).

Os fatores que desencadeiam o desenvolvimento da LP também podem afetar a cicatrização da pele por isso recomenda-se o uso de instrumentos de avaliação de feridas como: Bates-Jensen Wound Assessment Tool (BWAT) (18), e Pressure Ulcer Scale for Healing (PUSH) (35).

Como novas terapêuticas o destaque está para desbridamento (19), uso de laser (20), uso de solução de "ligustrazina" (34).

Em relação ao tratamento da LF, identificou-se somente um estudo, que recomenda o controle do sangramento, se esse existir. Seguido da limpeza da lesão com solução salina, aproximação do retalho avaliando o grau de perda tissular e a cor da pele ou do retalho, caso este esteja presente (tipo 1 pela classificação I STAP), avaliar e tratar a pele perilesional, escolher a cobertura apropriada para as características da lesão, reavaliando em $24 \mathrm{~h}$ a $48 \mathrm{~h}$ e por fim assegurar que a remoção da cobertura seja na direção do retalho e não contra ele (33). Em relação à escolha da cobertura, deve ser pensado no uso de produto não aderente que auxilie no processo de cicatrização e evite lesões nas trocas.

\section{Discussão}

A análise dos estudos mostrou predomínio de publicações no ano de 2016, sugerindo que a LF e LP são assunto de notoriedade contemporânea. Ademais, cinco artigos contemplados na revisão foram publicados na América do Sul, o que aponta, neste âmbito, uma lacuna na produção do conhecimento sobre a temática, a qual merece ser 
explorada, inclusive no Brasil, a fim de se revelar as diferentes realidades entre os diferentes países.

Diante da complexidade do tema, é necessário conhecer os fatores de risco para LF e LP a fim de evitá-las. Ao proceder à avaliação clínica da pele do idoso o enfermeiro deve levar em consideração as alterações pertinentes à idade, visto que há perda da elasticidade da pele devido à diminuição de colágeno e fibras elásticas, bem como diminuição da espessura das camadas da pele, tornando-a mais fina (47).

Em relação ao estado nutricional, estudo apresentou relação estatisticamente significante em relação ao IMC alterado, representando importante fator de risco para a ocorrência de LP (48). Assim como autores demonstram associação de precisão de LP no que se refere à albumina, quanto maior o nível de albumina, menor o risco de desenvolvimento de Lesão por Pressão, assim como os níveis de hemoglobina (49). Pesquisa (8) realizada em um hospital com idosos acima de 70 anos, avaliou 1412 pacientes com riscos de desenvolver LP em consonância à Escala de Braden, aponta que as intervenções nutricionais realizadas com maior frequência foram o fornecimento de suporte durante as refeições $(50,7 \%)$, alimentação especificamente desejada pelo paciente $(40,8 \%)$ e realização de rastreamento de desnutrição $(39,4 \%)$ e apenas um quarto dos pacientes foi encaminhado a um nutricionista; ressaltando que os profissionais de saúde precisam se conscientizar sobre a importância da nutrição no manejo de pacientes com LP.

Em relação à LF o The International Skin Tear Advisory Panel (ISTAP) apresentou três categorias de risco: Saúde geral (doença crônica e crítica, polifarmácia, cognitiva, sensorial, visual comprometimento auditivo e nutricional); Mobilidade (histórico de quedas, mobilidade prejudicada, dependência para atividades de vida diária e trauma mecânico) e a Pele (extremos de idade, pele frágil e rasgos anteriores da pele). A ISTAP também traduz o comportamento agressivo, agitado, hiperativo e inquieto como novas evidências para o risco de LF (50).

Estudo (51) demonstrou eficácia no reposicionamento de 4 horas e 6 horas em colchões de espuma viscoelásticos. Isso nos remete a importância não só do reposicionamento, mas também nos atentarmos às superfícies de apoio. Nesse ínterim, uma das pesquisas selecionadas para esta revisão utilizou uma tecnologia de monitoramento em relação ao posicionamento de idosos em instituição de longa permanência para facilitar a implementação da equipe de enfermagem do tratamento padrão para a prevenção LP. Desse modo, estimou a conformidade da equipe de enfermagem com o padrão de reposicionamento de cuidados antes e depois que os monitores visuais foram ativados para sinalizar a equipe demonstrando que o sistema melhorou significativamente $(\mathrm{P}=$ $0,0003)$ a conformidade com os padrões de reposicionamento a cada 2 horas (6).

Além disso, autores (52) comprovaram a eficácia clínica de curativos de espuma de silicone, aplicados em região sacra e calcânea na admissão de residentes em 40 lares de idosos australianos, e com alto risco de desenvolver uma lesão, promovendo a redução do risco relativo para $80 \%$.

Autores (53) avaliaram os planos de cuidados de 84 idosos destacando que as LF e LP foram reduzidas pelo uso de protetores de calcanhar e meias antiembólicas. Outro estudo ressalta a importância da utilização diária de emoliente para proteção da pele do idosos contra LF (5). O uso de um creme de barreira diário e/ou um hidratante diminuiu o número de lesões cutâneas, em comparação com outros produtos para a pele ou sem produtos.

Produtos emolientes estão disponíveis em hidratantes (pomadas, cremes e loções), óleos de banho, géis e substitutos de sabões. Os emolientes simples atuam absorvendo a umidade da pele e reduzindo a perda de água por evaporação, enquanto os emolientes que incluem substâncias adicionais conhecidas como umectantes (por exemplo, ureia) atuam atraindo ativamente a água da derme para a epiderme e compensando os seus níveis 
reduzidos de hidratantes naturais $(54,55)$.

Logo, outros autores (44) asseguram que a chave para prevenir a LF é reconhecer os pacientes com alto risco e implementar um protocolo de prevenção que incorpora algumas estratégias, quais sejam: identificar e remover possíveis fontes de lesão; considerar uso de roupas com as mangas compridas e calças para aqueles que repetidamente apresentam LF; usar cremes hidratantes hipoalergênicos emolientes (5) e limpadores de pele com PH neutro sem enxágue (sabonetes, lenços umedecidos e etc.); usar água morna para tomar banho; evitar usar produtos adesivos na pele frágil. Ressalta-se a importância da implementação de um pacote mínimo de cuidados para a efetivação destas ações (7). Além disso, acrescenta-se a importância da ingesta líquida adequada para hidratação, assim como incorpora a prevenção de quedas como parte da prevenção de lesões cutâneas ${ }^{44}$.

Ante o exposto, ressalta-se a relevância do Diagnóstico de Enfermagem (DE), visto que esse processo de interpretação é a base para a seleção das ações ou intervenções no que se refere à prevenção das lesões, bem como tratamento.

No tocante ao DE LP, um ano depois os autores validaram DE "Risco de Úlcera por pressão", elucidaram o DE "Lesão por Pressão", disponível na versão NANDA 2018 $2020^{56}$.

Logo, o termo LF foi selecionado por remontar à etiologia da ferida e assemelhar-se a outro termo cultural conhecido como LP, ficando estabelecida a nomenclatura LF segundo sua causa. Ainda, a LF não apresenta um diagnóstico próprio validado, sendo que até o momento, utilizamos o diagnóstico "integridade tissular prejudicada", que levam à perda da integridade tissular e comprometimento da sua função fisiológica, aproximando-se mais das características da LF (45).

Por fim, alguns autores (57) apresentam como destaque o uso de curativos/coberturas para o tratamento das LP, além disso, também foi observado sistema de medição digital e escalas. No que diz respeito às LF autores (49) distinguem o uso de cobertura não aderente (cobertura de silicone perfurado, malha de rayon impregnada com petrolatum ou AGE) para lesões secas ou exsudativas.

Atualmente, são diversas as opções de coberturas existentes no mercado. O enfermeiro deve avaliar as melhores opções de coberturas, considerando efetividade e recursos financeiros do paciente e/ou das unidades de saúde. A prática clínica baseada em evidências contribui para tomada de decisão, por meio de evidências científicas que assegurem recomendações para o uso.

Cabe pontuar que a prevalência de LF é subnotificada sendo, de fato, maior do que as LP. O envelhecimento da população traduz-se no reconhecimento de sua incidência está aumentando, uma vez que idosos têm pele frágil, o que aumenta o risco de sua ocorrência.

Assim, torna-se necessário que novos estudos sejam elucidados, visto à escassez de estudos que abordam a temática, principalmente as Lesões por Friç̧ão, visualizadas como limitadora do estudo, por isso, no intuito de fortalecer e embasar a prática assistencial do enfermeiro, permitindo a este profissional prevenir, diagnosticar e tratar LP e LF na população idosa, pautado em práticas cientificamente fundamentadas, passíveis de replicação no cenário de cuidado; a fim de que sua prática não seja pontuada no empirismo, fazem-se necessárias investigações futuras sobre a temática.

\section{Conclusão}

Os estudos legíveis para o tema proposto mostram-se escassos e prevaleceu o nível de evidência científica baixa, principalmente em relação à LF.

O maior quantitativo de publicações está centrado na identificação de fatores de risco de LP, sendo que as LF apresentam menor número. Em relação à prevenção das lesões, 
foram identificados estudos com idosos que abordavam somente LP, por mais que é sabido, que o idoso apresenta maior predisposição à LF e que essas, em comparação a LP, parecem ser irrelevantes, no entanto, são dolentes quando não tratadas adequadamente, podendo apresentar complicações graves como os quadros infecciosos.

Em se tratando de diagnóstico de enfermagem, há uma maior carência de publicações, sendo elegíveis somente três estudos. Isso nos faz refletir que a incidência e prevalências dessas lesões podem estar ligadas a não observância dos diagnósticos de enfermagem, visto que esses são fundamentais para elaboração do plano de assistência de enfermagem para prevenção.

Por fim, em relação ao tratamento, não diferente dos demais achados: pesquisas sobre LF em idosos ainda são incipientes. Isso nos remete o quão o enfermeiro precisa empoderar-se a fim de executar um atendimento de qualidade aos idosos com lesões, fundamentado em avaliação clínica baseada em evidência científica, assim como investir em produções científicas com melhores níveis de evidência.

\section{Referências}

1. Araújo LUA, Gama ZAS, Nascimento FLA, Oliveira HFV, Azevedo WM, Almeida Júnior HJB. Avaliação da qualidade da atenção primária à saúde sob a perspectiva do idoso. Ciênc. saúde coletiva. 2014; 19(8):3521-32.

2. Almeida AP, Bezerra ACP, Vieira GACM, Castro LHP, Costa NF. Úlceras por pressão na população idosa brasileira: Uma revisão sistemática. In: IV Congresso Internacional de Envelhecimento Humano. Anais CIEH. 2015; 2(1).

3. Botelho LLR, Cunha CCA, Macedo M. O método da revisão integrativa nos estudos organizacionais. Gestão e Sociedade. 2011; 5(11):121-36.

4. De Avila Soares, RS, Lima SBS, Eberhardt TD, Silveira LBTD, Pozzebon BR, Rodrigues LR, Alves PJP. Temperatura da pele de diferentes áreas corporais de idosos sem risco para lesões por pressão. Revista de Enfermagem da UFSM. 2020; 10 (e98): 1-19.

5. Moffat AK, Westaway KP, Apajee J, Frank O, Shute R, Weston C, Blacker N, Le Blanc VT, Kalisch Ellett LM, Pratt NL, Roughead EE. Impact of a patient-specific national programme aimed at increasing the use of emollient moisturisers to reduce the risk of skin tears: a longitudinal cohort study. BMJ Open. 2020; 10(10): e039579.

6. Yap TL, Kennerly SM, Ly K. Pressure Injury Prevention: Outcomes and Challenges to Use of Resident Monitoring Technology in a Nursing Home. J Wound Ostomy Continence Nurs. 2019; 46(3): 207-213.

7. Lavallée JF, Gray TA, Dumville JC, Cullum N. Preventing pressure injury in nursing homes: developing a care bundle using the Behaviour Change Wheel. BMJ Open. 2019; 9(6): e026639.

8. Eglseer D, Hödl M, Lohrmann C. Nutritional management of older hospitalised patients with pressure injuries. Int Wound J. 2019; 16(1): 226-232.

9. Rondinelli J, Zuniga S, Kipnis P, Kawar LN, Liu V, Escobar GJ. Hospital-Acquired Pressure Injury: RiskAdjusted Comparisons in an Integrated Healthcare Delivery System. Nurs Res. 2018; 67(1): 16-25.

10. Gray AT, Rhodes S, Atkinson RA, Rothwell K, Wilson P, Dumville JO, et al. Opportunities for better value wound care: a multiservice, cross-sectional survey of complex wounds and their care in a UK community population. BMJ Open. 2018; 8(3): 1-9.

11. Alderden JG, Pepper G. Pacientes críticos com necessidades especiais: pacientes geriátricos In: Hartjes TJ, ed. Currículo Básico AACN para Enfermagem de Alta Acuidade, Progressiva e Crítica. 7.ed. St Louis MO; Elsevier; 2018: 732-740.

12. Kaşđkçđ M, Aksoy M, Ay E. Investigation of the prevalence of pressure ulcers and patient-related risk factors in hospitals in the province of Erzurum: a cross-sectional study. J Tissue Viab 2018; 27(3): 13540.

13. Strazzieri-Pulido KC, González CV, Nogueira PC, Padilha KG, Santos VLC.Pressure injuries in critical patients: Incidence, patient-associated factors, and nursing workload. J nursmanage 2018; 27(2): 301-10.

14. Bermark S, Gerber AL, Philipsen PA, Skiveren J. Prevalence of skin tears in the extremities in inpatients at a hospital in Denmark. Int Wound J. 2018; 15(2): 212-7.

15. Han SH, Kim YS, Hwang J, Lee J, Song MR. Predictors of hospital-acquired pressure ulcers among older adult inpatients. J ClinNurs 2018; 27(19-20): 3780-6.

16. Kim CG, Seungmi P, WoonKo J, Sungho J. The relationship of subepidermal moisture and early stage 
pressure injury by visual skin assessment. J Tissue Viab. 2018; 27(3): 130-4.

17. Sardo PMG, Guedes JAD, Alvarelhão JJM, Machado PAP, Melo EMOP. Pressure ulcer incidence and Braden subscales: Retrospective cohort analysis in general wards of a Portuguese hospital. J Tissue Viab 2018; 27(2): 95-100.

18. Karahan A, Abbsogülu A, Isik SA, Cevik B, Saltan C, et al. Factors Affecting Wound Healing in Individuals With Pressure Ulcers: A Retrospective Study. Ostomy Wound Manage. 2018; 64(2): 32-9.

19. Chiang IH, Wang CH, TzengYS. Surgical treatment and strategy in patients with multiple pressure sores. I Wound J. 2018; 15(6): 900-8.

20. Taradaj J, Shay B, Dymarek R, Sopel M, Walewicz K, Beeckman D, et al. Effect of laser therapy on expression of angio- and fibrogenic factors, and cytokine concentrations during the healing process of human pressure ulcers. Int J MedSci. 2018; 15(11): 1105-12.

21. Smith IL, Brown S, McGinnis E, Briggs M, Coleman S, Dealey C, et al. Exploring the role of pain as an early predictor of category 2 pressure ulcers: a prospective cohort study. BMJ Open. 2017; 7(1): 01-13.

22. SkogestadIJ,Martinsen L,Børsting TE, Granheim I, Ludvigsen ES, Gay CL, Lerdal A. et al. Supplementing the Braden scale for pressure ulcer risk among medical inpatients: the contribution of self-reported symptoms and standard laboratory tests. J ClinNurs[Internet]. 2016; 26(1-2): 202-14.

23. Walker R, Huxley L, Juttner M, BurmeisterE,Scott J, Aitken LM. A pilot randomized controlled trial using prophylactic dressings to minimize sacral pressure injuries in high-risk hospitalized patients. Clin Nurs Res.2016; 26(4): 484-503.

24. Hanonu S, Karadag A. A prospective descriptive study to determine the rate and characteristics of and risk factors for the development of medical device- related pressure ulcers in Intensive Care Units. J Ostomy Wound Manage. 2016; 62:12-22.

25. Lannering C, Bravell ME, Midlov P, Ostgren CJ, Molstad S. Factors related to falls, weight-loss and pressure ulcers - more insight in risk assessment among nursing home residents. J ClinNurs[Internet]. 2016; 25(7-8): 940-50.

26. Mizokami F, Takahashi Y, Hasegawa K, Hattori H, Nishihara K, Endo H, et al. Pressure ulcers induced by drug administration: A new concept and report of four cases in elderly patients. J Dermatol. 2016; 43(4): 436-8.

27. Setoguchi Y, Ghaibeh AA, Mitani K, Abe Y, Hashimoto I, Moriguchi H. Predictability of pressure ulcers based on operation duration, transfer activity, and body mass index through the use of an alternating decision tree. J Med Invest. 2016; 63(34): 248-55.

28. Park KH, Choi H. Prospective study on Incontinence-Associated Dermatitis and its Severity instrument for verifying its ability to predict the development of pressure ulcers in patients with fecal incontinence. Int Wound J. 2016; 13:20-25.

29. Kang Y, Tzeng HM, Miller NA.Facilitycharacteristics and risk of developing pressure ulcers in US Nursing Homes. J Nurs Care Qual. 2016; 31:1: 9-16.

30. Athlin AM, Engström M, Gunningberg L, Bååth C. Heel pressure ulcer, prevention and predictors during the care delivery chain - when and where to take action? A descriptive and explorative study. Scand J Trauma, Res Emerg Med. 2016; 24(1): 134-40.

31. Kwong EW, Hung MS, Woo K. Improvement of pressure ulcer prevention care in private for-profit residential care homes: an action research study. BMC Geriatr. 2016; 16(1): 192-206.

32. Bååth C, EngströmM,GunningbergL,Athlin AM. Prevention of heel pressure ulcers among older patients - from ambulance care to hospital discharge: A multi-centre randomized controlled trial. Applied Nursing Research. 2016; 30:170-5.

33. Baranoski S, Leblanc K, Gloeckner M. CE: preventing, assessing, andmanagingskintears a clinicalreview. Am J Nurs. 2016; 116(11): 24-30.

34. Niu J, Han L, Gong F. Therapeutic effect of external application of Ligustrazine combined with holistic nursing on pressure sores. Med Sci Monit. 2016; 22: 2871-7.

35. Zeigler M, Smiley J, Ehrlich-Jones L, Moore JL. Use of the Pressure Ulcer Scale for Healing (PUSH) in Inpatient Rehabilitation: a case example. Rehabil Nurs. 2016; 41(4): 207-10.

36. Santos CT, Almeida MA, Lucena AF. Diagnóstico de Enfermagem risco de úlcera por pressão: validação de conteúdo. Rev Latinoam Enferm. 2016; 24: e2693.

37. Duim E, Sá FHC, Duarte YAO, Oliveira RCB, Lebrão ML. Prevalência e características das feridas em pessoas idosas residentes na comunidade. Revista da Escola de Enfermagem da USP. 2015; 49: 51-7.

38. Rasero L, Simonetti M, Falciani F, Fabbri C, Collini F, Dal Molin A. Pressure ulcers in older adults. Adv Skin Wound Care. 2015; 28(10): 461-4.

39. Lewin GF, Newall N, Alan JJ, Carville KJ, Santamaria NM, Roberts PA. Identification of risk factors associated with the development of skin tears in hospitalised older persons: a case-control study. Int Wound J. 2015; 13(6): 1246-51. 
40. Sardo P, Simões C, Alvarelhão J, Costa C, Simões CJ, Figueira J. Pressure ulcer risk assessment: retrospective analysis of Braden Scale scores in Portuguese hospitalised adult patients. J Clin Nurs. 2015; 24(21-22): 3165-76.

41. Sanada H, Nakagami G, Koyano Y, Iizaka S, SugamaJ.Incidence of skin tears in the extremities among elderly patients at a long-term medical facility in Japan: A prospective cohort study. Geriatr Gerontol Int. $2015 ; 15(8)$ : 1058-63.

42. Dijkstra A, Kazimier H, Halfens RJG. Using the Care Dependency Scale for identifying patients at risk for pressure ulcer. J Adv Nurs. 2015; 71(11): 2529-39.

43. Jaul E, Menczel J. A comparative, descriptive study of systemic factors and survival in elderly patients with sacral pressure ulcers. Int Wound J. 2015; 61(3): 20-6.

44. Iizaka S, Kaitani T, Nakagami G, Sugama J, Sanada H. Clinical validity of the estimated energy requirement and the average protein requirement for nutritional status change and wound healing in older patients with pressure ulcers: A multicenter prospective cohort study. Geriatr Gerontol Int. 2014; 15(11): 1201-9.

45. Strazzieri-Pulido KC, Santos, VLCG, Carville K. Cultural adaptation, content validity and inter-rater reliability of the "STAR Skin Tear Classification System". Rev Latino-am Enferm. 2015; 23(1): 155-61.

46. Melnyk BM, Fineout-Overholt E. Making the case for evidence-based practice. In: Melnyk BM, Fineout-Overholt E. Evidence-based practice in nursing \& healthcare. A guide to best practice. Philadelphia: Lippincot Williams \& Wilkins; 2005:3-24.

47. Fortes TML, Suffredini IB. Avaliação de pele em idoso: revisão da literatura. J Health Sci Inst. 2014; 32(1): 94-101.

48. Peixoto CA, Ferreira MBG, Felix MMS, Pires PS, Barichello E, Barbosa MH. Classificação de risco de desenvolvimento de lesões decorrentes do posicionamento cirúrgico. Rev. Latino-Am. Enfermagem. 2019; 27: e3117.

49. Fernandes LM, Silva L, Oliveira JLC, Souza VS, Nicola AL. Associação entre predição para lesão por pressão e marcadores bioquímicos. Rev. Rene. 2016; 17(4): 490-7

50. LeBlanc K, Baranoski S. Skin Tears: Finally Recognized. Advances in Skin \& Wound Care. 2017; 30(2): 62-63.

51. Gillespie BM, Chaboyer WP, McInnes E, Kent B, Whitty JA, Thalib L. Repositioning for pressure ulcer prevention in adults. Cochrane Database Syst Rev. 2014; 4: CD009958.

52. Santamaria N, Gerdtz M, Kapp S, Wilson L, Gefen A. A randomised controlled trial of the clinical effectiveness of multi-layer silicone foam dressings for the prevention of pressure injuries in high-risk aged care residents: The Border III Trial. Int Wound J. 2018; 15(3): 482-90.

53. Brimelow RE, Wollin JA. The impact of care practices and health demographics on the prevalence of skin tears and pressure injuries in aged care. J Clin Nurs. 2018; 27(7-8): 1519-28.

54. Wounds UK (2015) All Wales Guidance for the prevention and management of skin tears. Available online at: http://www.welshwoundnetwork.org/ files/8314/4403/4358/content_11623.pdf

55. LeBlanc K, Campbell KE, Wood E, Beeckman D. Best practice recommendations for the prevention and management of skin tears in aged skin. Wounds International 2018. Available from: http://www. woundsinternational.com

56. Santos CT, Bavaresco T, Almeida MA, Lucena AF. Lesão por pressão: novo diagnóstico submetido e aprovado à NANDA Internacional. VIII Simpósio do Processo de Enfermagem e I Simpósio Internacional do Processo de Enfermagem. Porto Alegre: HCPA, 2017.

57. Vieira CPB, Pinheiro DM, Luz MHBA, Araújo TME, Andrade EMLR. Tecnologias utilizadas por enfermeiros no tratamento de feridas. Ver Enferm UFPI. 2017; 6(1): 65-70. 